\title{
Shoulder Interfascial Plane Blocks as sole Anesthesia for Frozen Shoulder Manipulation
}

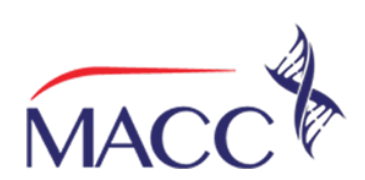

\author{
Syahrul Mubarak Danar Sumantri ${ }^{1 *}$
}

1. Department of Anesthesia and Critical Care, Siloam Hospitals Jember, Jember, Indonesia

*corresponding author

DOI: $10.55497 /$ majanestcricar.v38i3.196

\begin{abstract}
This is a case report of a successful awake frozen shoulder manipulation in a 62-year-old female under novel regional anaesthesia technique named shoulder interfascial plane blocks which cover suprascapular, subscapular, axillary, and lateral pectoral nerves. This combination of subomohyoid plane, subscapular plane, and PECS-1 blocks was performed before the procedure to cover the sensory dermatomal structure of the shoulder joint complex. For the resulting intra-procedure dynamic pain score 1 out of 10 without any additional sedatives nor rescue analgesia, shoulder interfascial plane blocks may be considered safe and effective.
\end{abstract}

Keywords: frozen shoulder; regional anesthesia; shoulder interfascial plane blocks 


\section{INTRODUCTION}

Manipulation under anesthesia is one of the frozen shoulder treatments that may promote immediate recovery of shoulder function and symptoms. ${ }^{1}$ Few clinicians has been opposed that sedation during manipulation for frozen shoulder is unable to ensure if any other structures than the joint capsule are damaged during manipulation. ${ }^{2}$

Shoulder interfascial plane blocks comprise three combined approaches, subomohyoid plane block to cover suprascapular nerve, subscapular plane block for subscapular and axillary nerves, and PECS-1 block to cover lateral pectoral nerve. Considering none of the reports about shoulder interfascial blocks as sole anesthesia for frozen shoulder manipulation were ever published before, this case report aimed to report a novel regional anesthesia technique that covered all target nerves of the shoulder, thus enabling frozen shoulder manipulation without any supplemental sedation or intravenous analgesic, yet still spared its motor function.

\section{CASE REPORT}

A 62-year-old lady who suffered frozen shoulder was scheduled for manipulation under awake regional anesthesia by patient's own request. Under strict aseptic technique, I administered shoulder interfascial plane blocks in supine position with a 3-13 MHz linear probe (Mindray M7, Shenzhen, China). The probe positions and needle directions for each block are shown in Fig. 1A. For the subomohyoid plane block, the probe was placed just superior to the clavicle with clear identification of brachial plexus, subclavian artery and omohyoid muscle inferior belly where a-5 $\mathrm{ml}$ ropivacaine $0.375 \%$ was deposited under (Fig. 1B). Subsequently, the probe was moved to the axial plane of shoulder where lesser trochanter of humerus and subscapularis muscle well identified. Later, a-15 $\mathrm{ml}$ ropivacaine $0.375 \%$ was deposited at ventral surface of subscapularis muscle (Fig. 1C). The last one was PECS-1 block with the probe positioned at midclavicular level, aligned and moved inferolaterally, until thoracoacromial artery seen sandwiched between pectoralis major and minor muscle where a-10 $\mathrm{ml}$ ropivacaine $0.375 \%$ was deposited within (Fig. 1D).

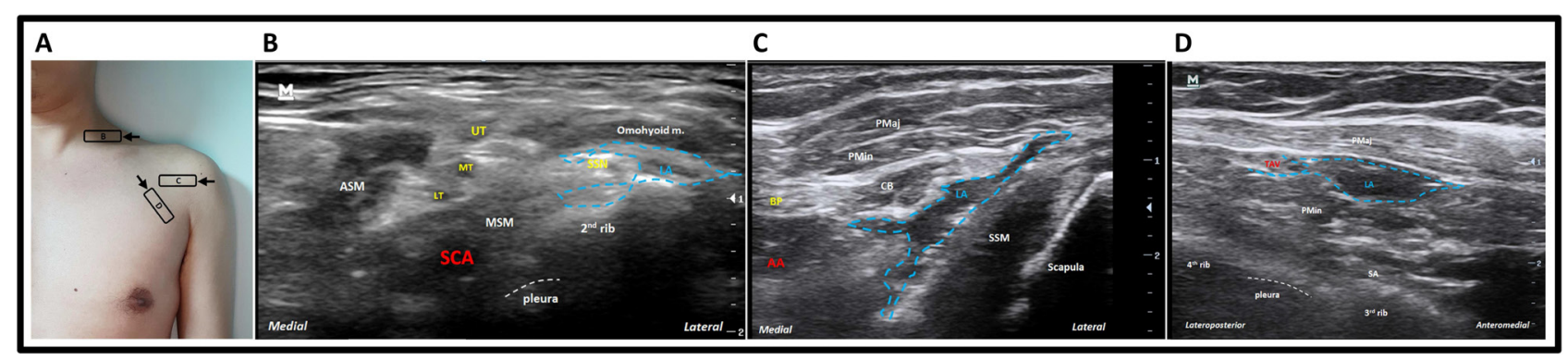

Figure 1. (A) Probe position and needle direction for subomohyoid plane block (B), subscapular plane block (C) and PECS-1 block (D). (B) Ultrasound image of local anaesthetic (LA) deposited under omohyoid muscle inferior belly. Suprascapular nerve (SSN) was seen immediately deep to omohyoid muscle, separating from brachial plexus : upper trunk (UT), middle trunk (MT) \& lower trunk (LT) laterally when probe swiped caudad. (C) Ultrasound image of local anaesthetic (LA) deposited anteriorly from the surface of subscapular muscle (SSM). The 3 layers of muscle at the anterior border of subscapular plane were coracobrachialis (CB), pectoralis minor (PMin), and pectoralis major (P.Maj) muscle. (D) Ultrasound image of the fascial plane between pectoral major (PMaj) and pectoral minor (Pmin) muscles, hidrodissected by local anesthetic (LA) where the pectoral branch of thoracoacromial vessel (TAV) lies. 


\section{DISCUSSION}

Throughout passive shoulder manipulation, the dynamic pain score was 1 out of 10 , yet the patient did not need any sedatives nor rescue analgesia. Sequentially after each maneuver of shoulder manipulation, the patient was able to actively move her upper arm allowing early identification of any possible mechanical injury that may occur to adjacent structures of the shoulder during manipulation. This report supports Dr.Price's and Dr.Sondekoppam's et al. statement that complete shoulder analgesia should include anterior structures as well, provided by blocking subscapular and lateral pectoral nerves with subscapular plane and PECS-1 blocks. ${ }^{3,4}$

\section{CONCLUSION}

Shoulder interfascial plane blocks effectively cover suprascapular, subscapular, axillary, and lateral pectoral nerves hence provide complete anesthesia of shoulder joint complex enabling painless shoulder manipulation. This novel regional anesthesia technique can be considered safe dan effective for awake frozen shoulder manipulation. Further study needed to define its clinical anatomy mechanism and to amplify its application in any shoulder procedure.

\section{REFERENCE}

1. Wang J, Huang $\mathrm{T}, \mathrm{Ma} H$. Manipulation under anesthesia for frozen shoulder in patients with and without non-insulin dependent diabetes mellitus. Int Orthop. 2010;34(8):1227-32.

2. Kraal T, Beimers L, The B, Sierevelt I. Shoulder \& Elbow Manipulation under anesthesia for frozen shoulders: outdated technique or well-established quick fix ? EFORT Open Rev. 2019;4(3):98-109.

3. Price D. How Many Nerves Supply the Shoulder? Reg Anesth Pain Med. 2018;43(3):2018.

4. Sondekoppam RV, Lopera-Velasquez L-M, Naik L, Ganapathy S. Subscapularis and sub-omohyoid plane blocks: an alternative to peripheral nerve blocks for shoulder analgesia. Br J Anaesth. 2016;117(6):831-2.

\section{CONFLICT OF INTEREST}

None. 